\title{
Synthesis and Characterization of Methoxy-bridged Bis(silylene)tungsten Complexes
}

\author{
R. Begum* and S. M. S. Islam \\ Department of Chemistry, Shahjalal University of Science and Technology, Sylhet-3114, \\ Bangladesh
}

Received1 July 2011, accepted in final revised form 27 October 2011

\begin{abstract}
Synthesis of two methoxy bridged bis(silylene) complexes $\left(\mathrm{Cp}^{\mathrm{Me} 4}\right) \mathrm{W}(\mathrm{CO})_{2}$ $\left\{\left(\mathrm{Et}_{2} \mathrm{Si}\right) \cdot \cdot \mathrm{OMe} \cdot \bullet\left(\mathrm{SiMe}_{2}\right)\right\}(3)$ and $\left(\mathrm{Cp}^{\mathrm{Me}}\right) \mathrm{W}(\mathrm{CO})_{2}\left\{\left(\mathrm{Et}_{2} \mathrm{Si}\right) \cdot \cdot \mathrm{OMe} \cdot \bullet\left(\mathrm{SiMe}_{2}\right)\right\}(\mathbf{4})$ have been synthesized. Both the compounds contain chiral tungsten center and formed via oxidative addition of an $\mathrm{Si}-\mathrm{H}$ bond to a coordinatively unsaturated metal center. The complexes were characterized by ${ }^{1} \mathrm{H},{ }^{13} \mathrm{C},{ }^{29} \mathrm{Si} \mathrm{NMR}$, IR and elemental analysis. A plausible mechanism of formation is also suggested.
\end{abstract}

Keywords: Coordinatively unsaturated; Chiral tungsten; Oxidative addition.

(c) 2012 JSR Publications. ISSN: 2070-0237 (Print); 2070-0245 (Online). All rights reserved. doi: $10.3329 /$ jsr.v4i1.7890

J. Sci. Res. 4 (1), 109-118 (2012)

\section{Introduction}

Silylene complexes have been proposed as reactive intermediates in a number of organosilicon transformations [1-7], however electrophilic nature of silylene ligands implies difficulties on there straightforward synthesis and consequent study on their properties. Efforts to synthesize silylene complexes resulted donor-stabilized silylene [814], donor stabilized bis(silylene) [15-19] and extremely donor stabilized sily(silyline) complexes [20]. A schematic diagram of the orbital interaction in donor stabilized silylene complex shows the role of filled d-orbitals of the transition metal atom in stabilizing the $\mathrm{sp}^{2}$ hybridized silylene by back donation and the contribution of the donor group (a Lewis base) in satisfying the extreme Lewis acidity of the silylene ligand (Fig. 1).

In 1977, Schimdt and Welz [21] first succeeded in isolating a donor-stabilized silylene complex (Fig. 2 a) by the reaction of $\mathrm{Fe}(\mathrm{CO})_{5}$ and $\mathrm{HSiMe}_{2} \mathrm{NEt}_{2}$. However the instability of this silylene complex above $-20^{\circ} \mathrm{C}$ prevented structural characterization. Ten years later

\footnotetext{
*Corresponding author: ruchy01@yahoo.com, rockshana-che@sust.edu
} 
Tilly et al. [22] and Zybill et al. [23] reported the first fully characterized donor stabilized silylene complexes, stabilized by electron rich metal center and bulky alkoxy substituents, respectively (Figs. 2b, c). Ogino et al. [13] reported synthesis of donor stabilized bis(silylene)iron complexes by photolysis of $\mathrm{Cp}(\mathrm{Me})_{5} \mathrm{Fe}(\mathrm{CO})_{2} \mathrm{SiMe}_{2} \mathrm{SiMe}(\mathrm{OMe})_{2}$ (Fig. 2 d).

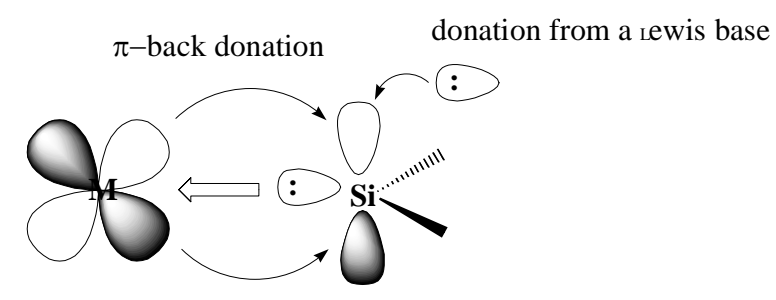

Fig. 1. Schematic diagram of the orbital interaction in donor stabilized silylene complex.<smiles>CCNC(C)(C)[Si](C)(C)C(=O)OC</smiles>

a

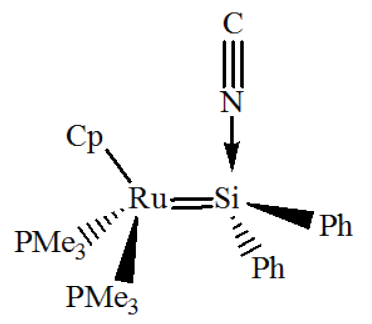

c

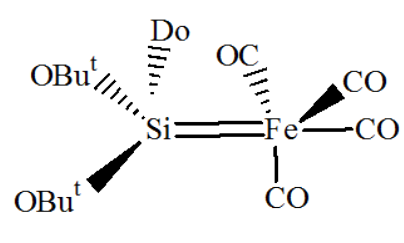

b<smiles>CO[Si]1(C)O[Si](C)(C)C([Te]C(=O)[O-])=[Si]1(C)C</smiles>

d

Fig. 2. Donor stabilized silylene complexes.

Because of their involvements in numerous transformation of organosilicon compounds, synthesis and study of complexes featuring silylene ligands are important to understand the mechanism of transformation. 
In this paper, synthesis of two methoxy bridged bis(silylene) complexes with chiral tungsten center are reported.

\section{Experimental}

\subsection{Methods and materials}

All manipulations were performed under a dry $\mathrm{N}_{2}$ or Ar atmosphere, using standard Schlenk tube techniques, a glove box, or a glove bag. Benzene, pentane, tetrahydrofuran (THF), and diethyl ether $\left(\mathrm{Et}_{2} \mathrm{O}\right)$ were dried by refluxing over sodium benzophenone ketyl followed by distillation under a dry $\mathrm{N}_{2}$ atmosphere, immediately prior to use. Diethylamine $\left(\mathrm{HNEt}_{2}\right)$ and $\mathrm{MeOH}$ were dried over $\mathrm{CaH}_{2}$ and $\mathrm{Mg}$, respectively and distilled under a nitrogen atmosphere before use. $\mathrm{LiAlH}_{4}$ was purchased from 'Wako Pure Chemical' and used as received.

$\mathrm{Cp}^{\mathrm{Me}} \mathrm{W}(\mathrm{CO})_{3} \mathrm{Me}_{4}$ and $\mathrm{ClSiEt}_{2} \mathrm{SiMe}_{2} \mathrm{Cl}$ [24] have been prepared according to the literature. Infrared spectra were obtained by using a HORIBA FT-200 spectrometer. ${ }^{1} \mathrm{H}$ NMR, ${ }^{13} \mathrm{C}$ NMR, ${ }^{29} \mathrm{Si}$ NMR, spectra were recorded on a Bruker ARX-300 Fourier transform spectrometer. Gas chromatography was carried out with a HITACHI 262-30 gas chromatograph with FID and a SE30/uniport B column (60/80 mesh carrier gas $\mathrm{N}_{2}$ flow rate: $30 \mathrm{mi} / \mathrm{min})$.

\subsection{Synthesis of $\mathrm{HEt}_{2} \mathrm{SiSiMe}_{2} \mathrm{NEt}_{2}$ [25]}

To a pentane solution (200 ml) of $\mathrm{ClSiEt}_{2} \mathrm{SiMe}_{2} \mathrm{Cl}(19 \mathrm{~g}, 0.09 \mathrm{~mol})$ at $0^{\circ} \mathrm{C}, \mathrm{HNEt}_{2}(16.1 \mathrm{~g}$, $0.22 \mathrm{~mol}$ ) was added with vigorous stirring. After $18 \mathrm{~h}$ of continuous stirring at room temperature, the reaction mixture was filtered through a Celite pad. Volatiles were removed from the filtered under reduced pressure. Pale yellow residue was added dropwise with vigorous stirring to a suspension of $\mathrm{LiAlH}_{4}(1.26 \mathrm{~g})$ in $\mathrm{Et}_{2} \mathrm{O}(20 \mathrm{ml})$ at $0^{\circ} \mathrm{C}$. After 30 min of stirring, the reaction mixture was filtered through a Celite pad repeatedly until a clear filtrated was obtained. The filtrate was then distilled under reduced pressure (bp. $107-110^{\circ} \mathrm{C} / 20$ torr). $\mathrm{HEt}_{2} \mathrm{SiSiMe}_{2} \mathrm{NEt}_{2}$ was obtained as an extremely moisture sensitive liquid (yield 59\%) and characterized by ${ }^{1} \mathrm{H}$ NMR. ${ }^{1} \mathrm{H}$ NMR $\left(\mathrm{C}_{6} \mathrm{D}_{6}, 300 \mathrm{MHz}\right)$

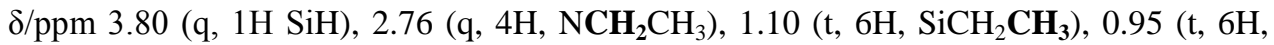
$\mathrm{NCH}_{2} \mathbf{C H}_{3}$ ), 0.7-0.6 (br, 4H, $\mathrm{SiCH}_{2} \mathrm{CH}_{3}$ ), 025 (s, 6H, SiCH${ }_{3}$ ).

\subsection{Synthesis of $\mathrm{HEt}_{2} \mathrm{SiSiMe}_{2} \mathrm{OMe}$ [25]}

To a benzene solution (10 ml) of $\mathrm{HEt}_{2} \mathrm{SiSiMe}_{2} \mathrm{NEt}_{2}(10 \mathrm{~g}, 0.046 \mathrm{~mol}) \mathrm{MeOH}(0.103 \mathrm{~mol})$ was added dropwise at $0^{\circ} \mathrm{C}$ with vigorous stirring. The reaction mixture was stirred at room temperature for $30 \mathrm{~min}$. Distillation of the mixture under atmospheric pressure gave the title compound as a moisture-sensitive liquid (0.029 mol, yield 63\%). The compound was characterized by ${ }^{1} \mathrm{H}$ NMR. ${ }^{1} \mathrm{H}$ NMR $\left(\mathrm{C}_{6} \mathrm{D}_{6}, 300 \mathrm{MHz}\right) \delta / \mathrm{ppm} 3.77$ (q, $\left.1 \mathrm{H} \mathrm{SiH}\right), 3.28$ 
(s, 3H, SiOMe), 1.08-1.00 (m, 6H, $\mathrm{SiCH}_{2} \mathbf{C H}_{3}$ ), 0.99-0.65 (m, 4H, SiCH $\mathrm{CH}_{3}$ ), 0.25(s, $\left.6 \mathrm{H}, \mathrm{SiCH}_{3}\right)$.

\subsection{Synthesis of $\left(\mathrm{Cp}^{\mathrm{Me4}}\right) \mathrm{W}(\mathrm{CO})_{3} \mathrm{Me}$}

To a mixture of $\mathrm{C}_{5} \mathrm{Me}_{4} \mathrm{H}_{2}$ (1.82ml, 0.012mol) and THF (100 ml) BuLi (1.4M solution in $n$-hexane, $8.22 \mathrm{ml}, 0.012 \mathrm{~mol})$ was added slowly via a syringe at room temperature. After 30 min of stirring $\mathrm{W}(\mathrm{CO})_{6}(4.57 \mathrm{~g}, 0.013 \mathrm{~mol})$ was added to the reaction mixture and then refluxed for $50 \mathrm{~h} . \mathrm{CH}_{3} \mathrm{I}(0.75 \mathrm{~g}, 0.012 \mathrm{~mol})$ was added dropwise via a syringe with continuous stirring of the reaction mixture. After $4 \mathrm{~h}$ stirring at room temperature, volatiles were removed from the reaction mixture using a rotary evaporator. The title compounds were obtained by sublimation of the residue at 0.3 torr $/ 70^{\circ} \mathrm{C}$ as orange crystal (0.004 mol, yield 33\%). The compound was characterized by ${ }^{1} \mathrm{H} N M R,{ }^{13} \mathrm{C}$ NMR, IR, and elemental analysis. ${ }^{1} \mathrm{H}$ NMR $\left(\mathrm{C}_{6} \mathrm{D}_{6}, 300 \mathrm{MHz}\right) \delta / \mathrm{ppm} 4.28\left(\mathrm{~s}, 1 \mathrm{H} \mathrm{C}_{5} \mathrm{Me}_{4} \mathrm{H}\right), 1.53(\mathrm{~s}, 6 \mathrm{H}$, $\left.\mathrm{C}_{5} \mathbf{M e}_{4} \mathrm{H}\right), 1.40\left(\mathrm{~m}, 6 \mathrm{H}, \mathrm{C}_{5} \mathbf{M e} \mathbf{e}_{4} \mathrm{H}\right), 0.38(\mathrm{~s}, 3 \mathrm{H}, \mathrm{W}-\mathbf{M e})$.

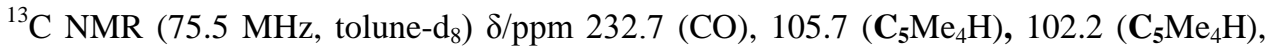
$87.8\left(\mathbf{C}_{5} \mathrm{Me}_{4} \mathrm{H}\right), 11.8\left(\mathrm{C}_{5} \mathbf{M e}_{4} \mathrm{H}\right), 11.6\left(\mathrm{C}_{5} \mathbf{M e}_{4} \mathrm{H}\right), 25.2$ (W-Me).

IR (KBr) v(CO) 1924, $2001 \mathrm{~cm}^{-1}$.

Anal. Calcd. for $\mathrm{C}_{13} \mathrm{H}_{16} \mathrm{WO}_{3}$ : C, 38.64; H, 3.98. Found: C, 38.71; H, 4.01.

\subsection{Synthesis of $\left(\mathrm{Cp}^{\mathrm{Me4}}\right) \mathrm{W}(\mathrm{CO})_{2}\left\{\left(\mathrm{Et}_{2} \mathrm{Si}\right) \bullet \bullet \mathrm{OMe} \bullet \cdot\left(\mathrm{SiMe}_{2}\right)\right\}(3)$}

A Pyrex sample tube $(10 \mathrm{~mm}$ o.d) with a ground glass joint was charged with $\left(\mathrm{Cp}^{\mathrm{Me} 4}\right) \mathrm{W}(\mathrm{CO})_{3} \mathrm{Me} \quad\left(0.12 \mathrm{~g}, 0.27 \times 10^{-3} \mathrm{~mol}\right)$ and $\mathrm{HEt}_{2} \mathrm{SiSiMe}_{2} \mathrm{OMe}\left(0.026 \mathrm{~g}, 1.74 \times 10^{-3}\right.$ $\mathrm{mol}$ ) and connected to a vacuum line via ground glass joint. About $5 \mathrm{ml}$ toluene was transferred to the sample tube by trap-to-trap distillation. The sample tube was then flame sealed under vacuum. The solution was irradiated for $30 \mathrm{~min}$ externally with a medium pressure Hg arc lamp (Ushio UV-450) placed in a water cooled quartz jacket. During irradiation, the sample tube was immersed in ice water. The distance from the light source to the sample was ca. $4 \mathrm{~cm}$. After irradiation, the reaction mixture was transferred into a Schlenk tube, concentrated, and cooled to $-18^{\circ} \mathrm{C}$ in a refrigerator to give the title compound as air and moisture sensitive needle-type crystals (0.22 g, yield $81 \%)$. The compound was characterized by ${ }^{1} \mathrm{H}$ NMR, ${ }^{13} \mathrm{C}$ NMR, ${ }^{29} \mathrm{Si}$ NMR, High resolution mass, and elemental analysis. ${ }^{1} \mathrm{H}$ NMR $\left(\mathrm{C}_{6} \mathrm{D}_{6}, 300 \mathrm{MHz}\right) \delta / \mathrm{ppm} 4.68\left(\mathrm{~s}, 1 \mathrm{H} \mathrm{C}_{5} \mathrm{Me}_{4} \mathrm{H}\right), 2.82$ (s, 6H, SiOMe), 1.97 (s, 6H, C ${ }_{5} \mathbf{M e}_{4} \mathrm{H}$ ), 1.96 (s, 6H, C ${ }_{5} \mathbf{M e}_{4} \mathrm{H}$ ), 1.02 (br, 6H, $\mathrm{SiCH}_{2} \mathbf{C H}_{3}$ ), 1.00 (br, 4H, SiCH $\mathrm{CH}_{3}$ ), 0.58 (br, 6H, $\mathrm{SiCH}_{3}$ ).

${ }^{13} \mathrm{C}$ NMR (75.5 MHz, tolune-d $\left.d_{8}\right) \delta / p p m 235.9(\mathbf{C O}), 107.5\left(\mathbf{C}_{5} \mathrm{MeH}_{4}\right), 105.1\left(\mathbf{C}_{5} \mathrm{Me}_{4} \mathrm{H}\right)$, $93.0\left(\mathbf{C}_{5} \mathrm{MeH}_{4}\right), 57.4$ (SiOMe), $16.8\left(\mathrm{C}_{5} \mathbf{M e H}_{4}\right), 14.7\left(\mathrm{C}_{5} \mathbf{M e H}_{4}\right), 18.5,16.4$ (SiEt), 13.1 (SiMe).

${ }^{29} \mathrm{Si}$ NMR (59.6 MHz, tolune- $d_{8}$, DEPT) $\delta / \mathrm{ppm} 114.0\left({ }^{1} J_{\mathrm{Si}-\mathrm{W}}=97.8 \mathrm{~Hz}\right), 103.2\left({ }^{1} J_{\mathrm{Si}-\mathrm{W}}=\right.$ 100.1Hz).

IR $\left(\mathrm{C}_{6} \mathrm{D}_{6}\right) \vee(\mathrm{CO}) 1899,1830 \mathrm{~cm}^{-1}$. 
Mass (EI, $70 \mathrm{eV}) \mathrm{m} / \mathrm{z} 536$ ( $\left.\mathrm{M}^{+}, 100\right), 507$ (M+-Et, 75), 476 ( $\mathrm{M}^{+}$-Et-OMe, 30).

High resolution mass spectrum: Calculated for $\mathrm{C}_{18} \mathrm{H}_{32} \mathrm{Si}_{2} \mathrm{WO}_{3}$ : 536.1400; observed: 536.1407.

Anal. Calcd. for $\mathrm{C}_{18} \mathrm{H}_{32} \mathrm{Si}_{2} \mathrm{WO}_{3}$ : C, 40.24; H, 5.96. Found: C, 40.82; H, 6.16.

\subsection{Synthesis of $\left(C p^{M e}\right) W(C O)_{2}\left\{\left(E t_{2} S i\right) \cdot \bullet O M e \bullet\left(S_{M} M e_{2}\right)\right\}(4)$}

The title compound $\mathbf{4}$ was synthesized by a similar method described for $\mathbf{3}$, using $\left(\mathrm{Cp}^{\mathrm{Me}}\right) \mathrm{W}(\mathrm{CO})_{3} \mathrm{Me}\left(0.045 \mathrm{~g}, 0.12 \times 10^{-3} \mathrm{~mol}\right)$ and $\mathrm{HEt}_{2} \mathrm{SiSiMe}_{2} \mathrm{OMe}\left(0.066 \mathrm{~g}, 0.37 \times 10^{-3}\right.$ mol). Complex 4 was obtained as air and moisture sensitive needle-type yellow crystals (0.036 g, yield 85\%). The compound was characterized by ${ }^{1} \mathrm{H}$ NMR, ${ }^{13} \mathrm{C}$ NMR, ${ }^{29} \mathrm{Si}$ NMR, High resolution mass, and elemental analysis.

${ }^{1} \mathrm{H}$ NMR (300MHz, tolune-d $\mathrm{d}_{8}$ ) $/ \mathrm{ppm} 4.81$ (ddd, 2H, $\mathrm{C}_{5} \mathrm{MeH}_{4}$ ), 4.66 (ddd, 2H, ${ }_{5} \mathrm{MeH}_{4}$ ), 2.81(s, 3H, SiOMe), 1.93 (s, 3H, $\mathrm{C}_{5} \mathbf{M e H}_{4}$ ), 1.11-0.30 (br, 11H, SiEt), 0.31 (br, 6H, SiMe).

${ }^{13} \mathrm{C}$ NMR (75.5 MHz, tolune-d $\left.{ }_{8}\right)$ d/ppm 228.5 (CO), $105.3\left(\mathbf{C}_{5} \mathrm{MeH}_{4}\right), 88.8\left(\mathbf{C}_{5} \mathrm{MeH}_{4}\right)$, 52.4 (SiOMe), $16.8\left(\mathrm{C}_{5} \mathbf{M e H}_{4}\right), 10.0\left(\mathrm{C}_{5} \mathbf{M e H}_{4}\right), 21.3,14.5$ (SiEt), 7.9 (SiMe).

${ }^{29} \mathrm{Si}$ NMR (59.6 MHz, tolune-d $\left.\mathrm{d}_{8}\right) \delta / \mathrm{ppm} 114.3\left({ }^{1} J_{\mathrm{Si}-\mathrm{W}}=96.6 \mathrm{~Hz}\right), 102.3\left({ }^{1} J_{\mathrm{Si}-\mathrm{W}}=98.9 \mathrm{~Hz}\right)$. IR $\left(\mathrm{C}_{6} \mathrm{D}_{6}\right) \vee(\mathrm{CO}) 1907,1839 \mathrm{~cm}^{-1}$.

Mass (EI, 70 eV) m/z 494 (M+, 21), 465 (M+Et, 12), 323 ( M $^{+}$-Et-SiOMeSi-CO, 45).

High resolution mass spectrum: Calculated for $\mathrm{C}_{15} \mathrm{H}_{26} \mathrm{Si}_{2} \mathrm{WO}_{3}$ : 494.0896; observed: 494.0919.

Anal. Calcd. for $\mathrm{C}_{15} \mathrm{H}_{26} \mathrm{Si}_{2} \mathrm{WO}_{3}$ : C, 36.43; H, 5.30. Found: C, 36.63; H, 5.61.

\section{Results and Discussions}

Irradiation of a solution of $\left(\mathrm{Cp}^{\mathrm{Me} 4}\right) \mathrm{W}(\mathrm{CO})_{3} \mathrm{Me}$ and $\mathrm{HEt}_{2} \mathrm{SiSiMe}_{2} \mathrm{OMe}$ produces a methoxy-bridged bis(silylene) tungsten complex 3 (Eq. 1).
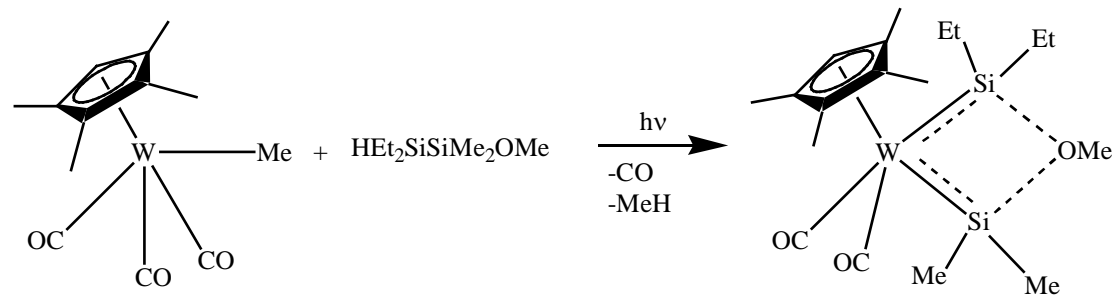

(eq. 1)

${ }^{1} \mathrm{H}$ NMR spectrum of 3 (Fig. 3) shows a singlet signal for $\mathrm{C}_{5} \mathrm{Me}_{4} \mathbf{H}$ proton at $4.68 \mathrm{ppm}$. Signals for 4 methyl groups of $\mathrm{Cp}^{\mathrm{Me} 4}$ appear as two singlets at 1.97 and 1.96. Appearance of two singlets for two sets of diastereotopic methyls instead of four signals indicated very fast conversion of chirality. Signals of two methyls and two ethyls groups on silicon atom 


\section{Synthesis and Characterization}

appear as broad signals around 1.1-0.3 ppm because of fast inter-conversion behavior due to silylene-methyl and silylene- ethyl group exchange [24]. For methoxy group, a singlet appears at $2.81 \mathrm{ppm} .{ }^{13} \mathrm{C}$ NMR spectrum of $\mathbf{3}$ showing only one singlet for two diastereotopic carbonyls also indicates fast conversion of chirality.

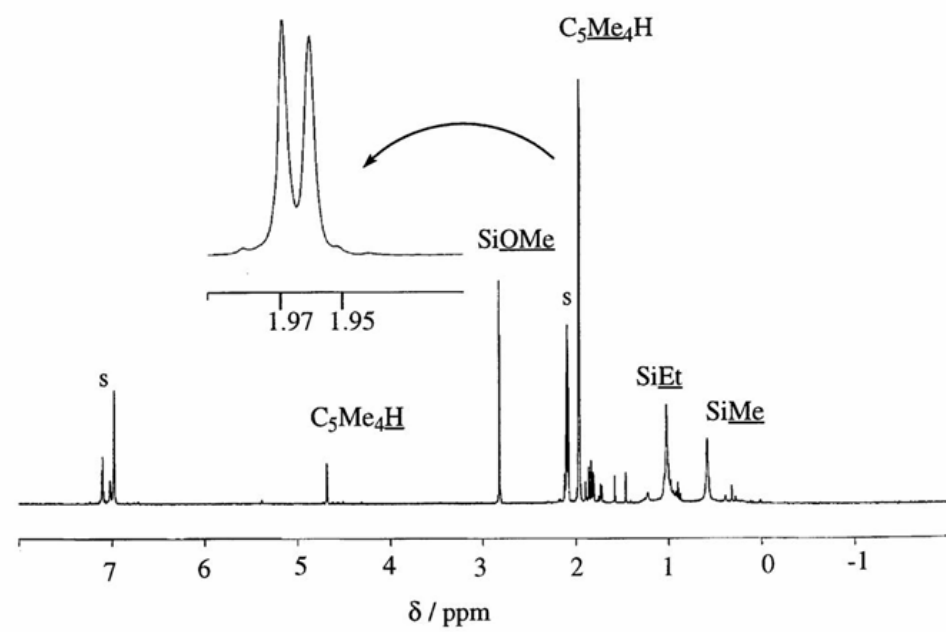

Fig. 3. ${ }^{1} \mathrm{H}$ NMR spectrum of $\left(300 \mathrm{MHz}\right.$, tolune- $\left.d_{8}\right)\left(\mathrm{Cp}^{\mathrm{Me} 4}\right) \mathrm{W}(\mathrm{CO})_{2}\left\{\left(\mathrm{Et}_{2} \mathrm{Si}\right) \bullet \mathrm{OMe} \bullet\left(\mathrm{SiMe}_{2}\right)\right\}(3)$.

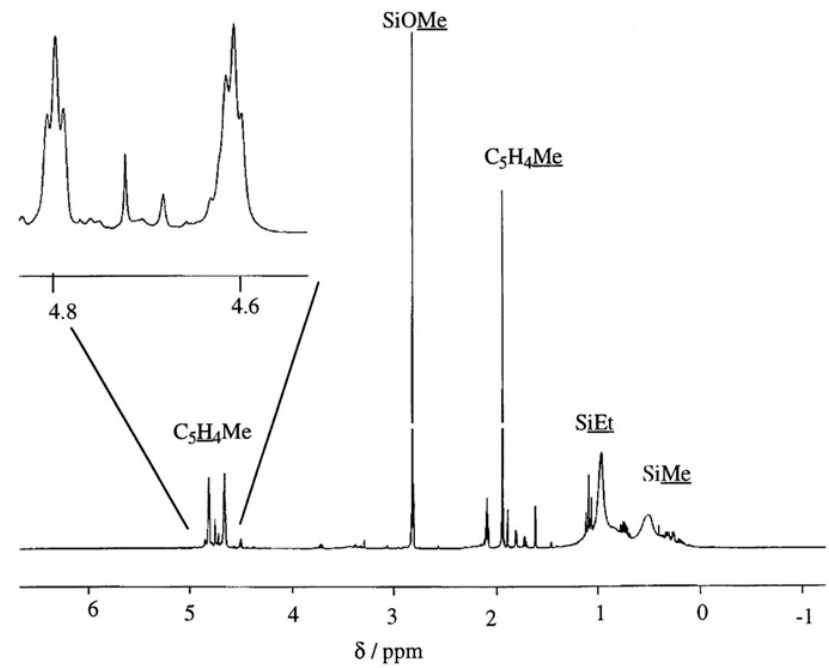

Fig. 4. ${ }^{1} \mathrm{H}$ NMR spectrum of (300MHz, tolune- $\left.d_{8}\right)\left(\mathrm{Cp}^{\mathrm{Me}}\right) \mathrm{W}(\mathrm{CO})_{2}\left\{\left(\mathrm{Et}_{2} \mathrm{Si}\right) \cdot \bullet \mathrm{OMe} \cdot \bullet\left(\mathrm{SiMe}_{2}\right)\right\}(4)$.

Irradiation of a solution of $\left(\mathrm{Cp}^{\mathrm{Me}}\right) \mathrm{W}(\mathrm{CO})_{3} \mathrm{Me}$ and $\mathrm{HEt}_{2} \mathrm{SiSiMe}_{2} \mathrm{OMe}$ produces a methoxy- bridged bis(silylene) tungsten complex 4 (Eq. 2). 

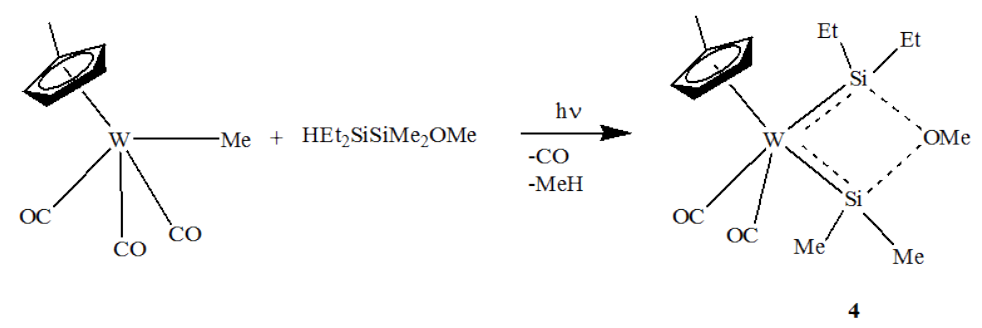

${ }^{1} \mathrm{H}$ NMR spectrum of 4 (Fig. 4) shows two multiplets for $\mathrm{C}_{5} \mathrm{MeH}_{4}$ protons at 4.83 and $4.67 \mathrm{ppm}$. Appearance of two sets of signals for two sets of diastereotopic protons instead of four sets signals indicated very fast conversion of chirality. Signals of two methyls and two ethyls groups on silicon atom appear as broad signals around 1.11-0.3 ppm because of fast inter-conversion behavior due to silylene-methyl and silylene-ethyl group exchange [25]. For methoxy group, a singlet appears at $2.77 \mathrm{ppm} .{ }^{13} \mathrm{C}$ NMR spectrum of $\mathbf{4}$ shows only one singlet for two diastereotopic carbonyls which also indicates fast conversion of chirality.

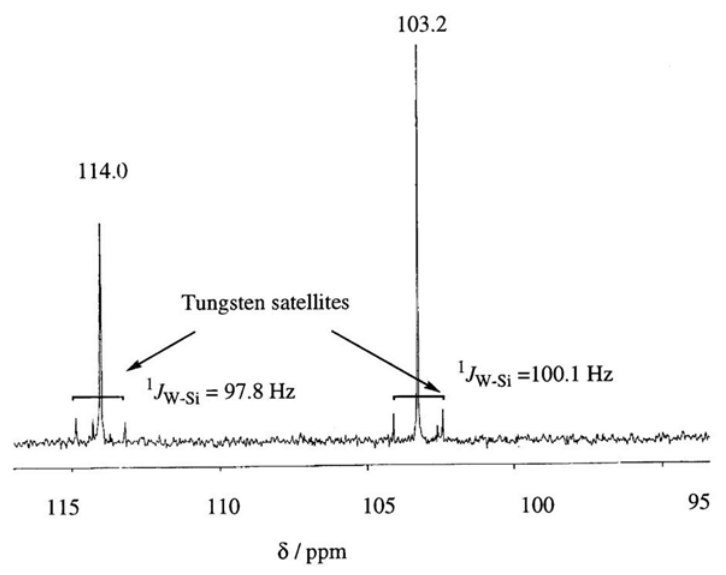

Fig. 5. ${ }^{29} \mathrm{Si}$ NMR $\left(59.6 \mathrm{MHz}\right.$, tolune- $\left.d_{8}\right)$ spectrum of $\left(300 \mathrm{MHz}\right.$, tolune- $\left.d_{8}\right)\left(\mathrm{Cp}^{\mathrm{Me} 4}\right) \mathrm{W}(\mathrm{CO})_{2}\left\{\left(\mathrm{Et}_{2} \mathrm{Si}\right) \bullet\right.$ Ome••(SiMe $)\}(4)$.

Complex 3 (Fig. 5) shows two ${ }^{29}$ Si NMR signals at 114.0 ppm. In case of 4 (Fig. 6) two signals appear at 114.3 and $102.3 \mathrm{ppm}$. Both spectra show tungsten satellites (183W; nuclear spin, $I=1 / 2$; natural abundance, $14.4 \%$ ) which are very useful indication between bonding of tungsten and silicon in bis(silylene) complex [26]. The coupling constants ${ }^{1} J_{\mathrm{Si}}$ w (97.8 and $100.1 \mathrm{~Hz}$ for 3 , and 96.6 and $98.9 \mathrm{~Hz}$ for 4 ) directly indicate the contribution of $s$ orbital in W-Si bonding. The large values of ${ }^{1} J_{\mathrm{Si}-\mathrm{W}}$ which have been observed for the studied complexes imply unsaturated nature in Si-W bonds [17]. 


\section{Synthesis and Characterization}

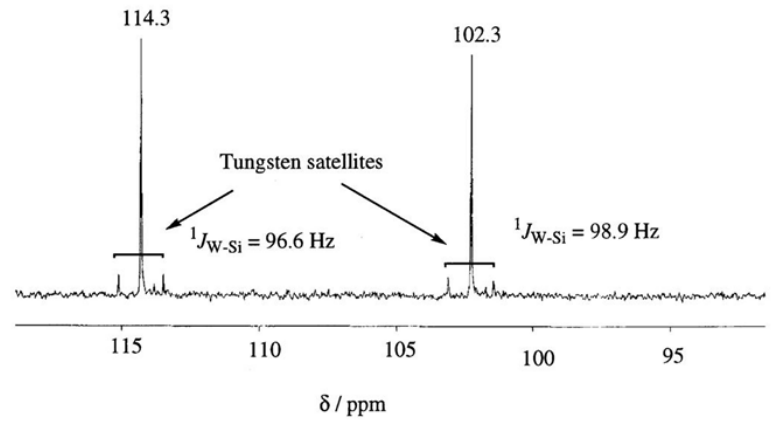

Fig. 6. ${ }^{29} \mathrm{Si}$ NMR $\left(59.6 \mathrm{MHz}\right.$, tolune- $\left.d_{8}\right)$ spectrum of $\left(300 \mathrm{MHz}\right.$, tolune- $\left.d_{8}\right)\left(\mathrm{Cp}{ }^{\mathrm{Me}}\right) \mathrm{W}(\mathrm{CO})_{2}\left\{\left(\mathrm{Et}_{2} \mathrm{Si}\right) \bullet\right.$ $\left.\mathrm{OMe} \bullet\left(\mathrm{SiMe}_{2}\right)\right\}(4)$.

\subsection{Mechanism of the reaction}

The proposed mechanism for the photolysis of $(\mathrm{Cp} /) \mathrm{W}(\mathrm{CO})_{3} \mathrm{Me}$ in presence $\mathrm{HEt}_{2} \mathrm{SiSiMe}_{2} \mathrm{OMe}$ is shown in Scheme-1.

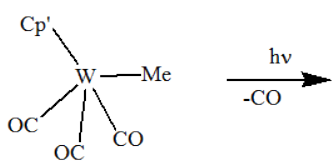

A

$\mathrm{Cp}^{\prime}=\mathrm{C}_{5} \mathrm{Me}_{4} \mathrm{H}, \mathrm{C}_{5} \mathrm{H}_{4} \mathrm{Me}$

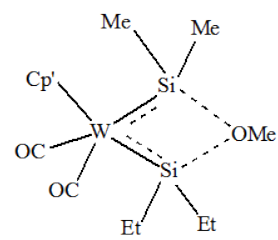

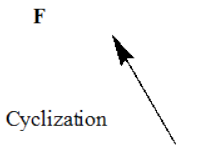

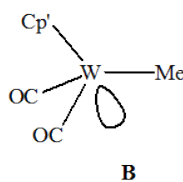

B

16 electron complex

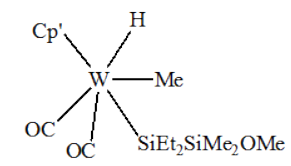

$\mathrm{HEt}_{2} \mathrm{SiSiMe}_{2} \mathrm{OMe}$

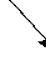

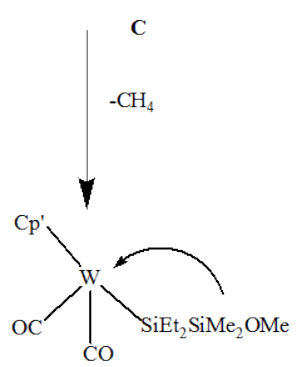

D 
The proposed mechanism is similar as reported by Wada et al. [17] and Ogino et al. [13]. The mechanism can be described as i) photochemical elimination of a carbonyl group results in a 16- electron complex $\mathbf{B}$, ii) Oxidative addition of a $\mathrm{Si}-\mathrm{H}$ bond of $\mathrm{HSiEt}_{2} \mathrm{SiMe}_{2} \mathrm{OMe}$ to give an 18 electron intermediate $\mathbf{C}$, iii) reductive elimination of $\mathrm{CH}_{4}$ to give a 16 electron complex $\mathbf{D}$, iv) 1,2-silyl group migration, and v) cyclization to form a chiral methoxy- bridged bis(silylene) tungsten complex.

\section{Conclusion}

Synthesis of two methoxy bridged bis(silylene) complexes with chiral tungsten center are reported via oxidative addition of an $\mathrm{Si}-\mathrm{H}$ bond to a coordinatively unsaturated Tungsten center. The complexes were characterized by $\mathrm{IR},{ }^{1} \mathrm{H},{ }^{13} \mathrm{C},{ }^{29} \mathrm{Si} \mathrm{NMR}$ and Elemental analysis. A plausible mechanism of formation is also suggested.

\section{Acknowledgment}

We thank Professor Dr. Hiroshi Ogino, Department of Chemistry, Tohoku University, Tohoku, Japan for his kind permission to conduct all the measurements. We are grateful to Keiji Ueno for his encouraging support and discussion.

\section{References}

1. I. Ogima, S. Inaba, T. Kogure, and Y. Nagai, J. Organomet. Chem. 55, C7 (1973). http://dx.doi.org/10.1016/S0022-328X(00)84018-8

2. H. Okinoshima, K. Yamamoto, and M. Kumada, J. Am. Chem. Soc. 94, 9263, (1972). http://dx.doi.org/10.1021/ja00781a066

3. H. Sakurai, Y. Kamiyama, and Y. Nakadaira, J. Am. Chem. Soc. 99, 3879 (1977). http://dx.doi.org/10.1021/ja00453a078

4. H. Nakadaira, T. Kabayashi, and H. Sakurai, J. Organomet. Chem. 165, 399 (1979). http://dx.doi.org/10.1016/S0022-328X(00)82209-3

5. H. Sakurai, Y. Kamiyama, and Y. Nakadaira, J. Organomet. Chem. 184, 13, (1977). http://dx.doi.org/10.1016/S0022-328X(00)94359-6

6. G. Thum and W. Malisch, J. Organomet. Chem, 264 C5, (1984). http://dx.doi.org/10.1016/0022-328X(84)85086-X

7. H. Kang, D. B. Jacobson, S. K. Shin, J. L. Beauchamp, and T. M. Bowere, J. Am. Chem. Soc. 108, 5668, (1986). http://dx.doi.org/10.1021/ja00279a002

8. D. A. Straus, and T. D. Tilly, J. Am. Chem. Soc. 109, 5892 (1987). http://dx.doi.org/10.1021/ja00253a062

9. D. A. Straus, C. Zhang, G. E. Quimbita, S. D. Grumbine, R. H. Hein, T. D. Tilly, A. L. Rheingold, and S. J. Geib, J. Am. Chem. Soc. 112, 2673 (1990). http://dx.doi.org/10.1021/ja00177a053

10. C. Zybill, and G. Muller, Organometallics, 7, 1368, (1988). http://dx.doi.org/10.1021/om00096a022

11. C. Zybill and G. Muller, Angrew. Chem. Int. Ed. Engl. 27. 583, (1988). http://dx.doi.org/10.1002/anie.198805831

12. C. Zybill, D. L. Wilkinson, C. Lewis, and G. Muller, Angew. Chem. Int. Ed. Engl. 28. 203, (1989). http://dx.doi.org/10.1002/anie.198902031 


\section{Synthesis and Characterization}

13. K. Ueno, H. Tobita, M. Shimoi, and H. Ogino, J. Am. Chem. Soc. 110, 4092, (1988). http://dx.doi.org/10.1021/ja00220a089

14. H. Tobita, K. Ueno, M. Shimoi, and H. Ogino, J. Am. Chem. Soc. 112, 3415, (1990). http://dx.doi.org/10.1021/ja00165a026

15. T. Takeuchi, H. Tobita, and H. Ogino, Organometallics 10, 835, (1991). http://dx.doi.org/10.1021/om00050a006

16. K. Ueno, S. Eto, K. Endo, H. Tobita, S. Inomata, and H. Ogino, Organometallics 13, 3309, (1994). http://dx.doi.org/10.1021/om00020a049

17. H. Tobita, H. Wada, K. Ueno, and H. Ogino, Organometallics 13, 2545, (1994). http://dx.doi.org/10.1021/om00019a003

18. M. Okazaki, H. Tobita, and H. Ogino, Chem. Lett. 437, (1997). http://dx.doi.org/10.1246/cl.1997.437

19. K. Ueno, A. Matsuka, and H. Ogino, Organometallics 16, 5023, (1997). http://dx.doi.org/10.1021/om970609h

20. K. Ueno, S. Asami, N. Watanabe, and H. Ogino, Organometallics 21, 1326, (2002). http://dx.doi.org/10.1021/om020288w

21. G. Schmid, and E. Welz, Angew Chem., Int. Ed. Engl., 16, 785 (1977). http://dx.doi.org/10.1002/anie.197702491

22. D. A. Straus, T.D. Tilley, A. L. Rheingold, and S. J. Geib, J. Am. Chem. Soc. 109, 5872 (1987). http://dx.doi.org/10.1021/ja00253a062

23. C. Zybill, and G. Muller, Angew. Chem. 26, 669 (1987). http://dx.doi.org/10.1002/anie.198706691

24. K. Ueno, A. Masuko, and H. Ogino, Organometallics 16, 5023 (1997). http://dx.doi.org/10.1021/om970609h

25. A. Masuko, M. Sc. Thesis, Tohoku University, Japan, (1995).

26. S. Schmitzer, U. Weis, H. Kab, W. Buchner, W. Malisch, T. Polzer, U. Posset, and W. Kiefer, Inorg. Chem., 32, 303 (1993). http://dx.doi.org/10.1021/ic00055a013 